\title{
Scalable TCP: Better Throughput in TCP Congestion Control Algorithms on MANETs
}

\author{
M.Jehan \\ Associate Professor, Department of Computer Science, \\ D.J.Academy for Managerial Excellence, \\ Coimbatore, India
}

\begin{abstract}
In the modern mobile communication world the congestion control algorithms role is vital to data transmission between mobile devices. It provides better and reliable communication capabilities in all kinds of networking environment. The wireless networking technology and the new kind of requirements in communication systems needs some extensions to the original design of TCP for on coming technology development. This work aims to analyze some TCP congestion control algorithms and their performance on Mobile Ad-hoc Networks (MANET). More specifically, we describe performance behavior of BIC, Vegas and Scalable TCP congestion control algorithms. The evaluation is simulated through Network Simulator (NS2) and the performance of these algorithms is analyzed in the term of efficient data transmission in wireless and mobile environment.
\end{abstract}

Keywords- TCP Congestion Control Algorithms; MANET; BIC; Vegas; Scalable TCP.

\section{INTRODUCTION}

Ad hoc network is a temporary network connection for a specific purpose (such as transferring data from one computer to another) in wireless networks. It is self organizing networks, which all end nodes are act as routers or data user. It improves the efficiency of fixed and mobile internet access and enables new applications for public. A Mobile Ad hoc Networks (MANET) consists of a set of mobile hosts within the communication range and exchange data among themselves without using any pre-existing infrastructure. MANET nodes are typically distinguished by their limited power, processing and memory resources as well as high degree of mobility. In such networks, the wireless mobile nodes may dynamically enter the network as well as leave the network. Due to the limited transmission range of wireless network nodes, multiple hops are usually needed for a node to exchange information to other node.

MANETs uses in the disparate situations such as moving battlefield communications to disposable sensors which are dropped from high altitude and dispersed on the ground for hazardous materials detection. The civilian applications include simple scenarios such as people at a conference in a hotel their laptops comprise a temporary Ad hoc Networks to more complicated scenarios such as highly mobile vehicles on the highway which form an Mobile Ad hoc Networks in order to provide traffic monitoring system.

\author{
Dr. G.Radhamani \\ Professor \& Director, Department of Computer Science, \\ Dr.G.R.Damodaran College of Science, \\ Coimbatore, India
}

This paper is entirely devoted to evaluating the Control Window (cwnd), Round Trip Delay Time (rtt) and Throughput using the TCP BIC, Vegas and Scalable TCP congestion control algorithms in the wireless networks.

\section{BACKGROUND WORK}

\section{A. Congestion Control in Transmission Control Protocol Algorithms}

TCP (Transmission Control Protocol) is a set of rules (protocol) used along with the Internet Protocol (IP) to send data in the form of message units between computers over the Internet. It operates at a higher level, concerned only with the two end systems. In particular, TCP provides reliable, ordered delivery of a stream of bytes from a program on one computer to another program on another computer. Among its other management tasks, TCP controls segment size, flow control, the rate at which data is exchanged, and network traffic congestion.

TCP can support the mechanisms of flow and congestion control for reliable data transmission. Due to the unconstrained movement of the mobile nodes, TCP is unable to notice network congestion or link down to activate related controls on the MANET [3]. The standard congestion control mechanism of the TCP is not able to handle the special properties of a shared wireless multi-hop channel well. In particular, the frequent changes of the network topology and the shared nature of the wireless channel create some critical issues [7].

It provides consistent end-to-end delivery of data over wired networks, several recent studies have indicated that TCP performance degrades significantly in MANET [10] [11]. In [16], TCP-F is proposed to overcome the TCP false reaction towards route failures in MANETs. In [17] the simulation shows that the route change results in link disconnections, which reduces TCP throughput.

TCP Vegas was the first attempt to depart from the lossdriven paradigm of the TCP by introducing a mechanism of congestion detection before packet losses [12].

Using TCP more computers are interconnected to increase data transaction between users rapidly. The MIMD and PIPD protocols developed and provides better throughput for the wireless networks [15], [19] and [20]. 
So, this experiment on the existing TCP congestion control algorithms and its performance on MANET will be very useful to design new algorithms and create innovative approach for mobile wireless communication in the point of reducing data loss during transmission.

\section{B. Congestion Control Algorithms For Evaluationons}

Congestion is characterized by delay and loss of packets in delivery. In TCP, congestion is said to have occurred when the sender receives three duplicate acknowledgments (dupacks) or when a timeout (packet loss) occurs, resulting in wastage of resources. Congestion Control and Congestion Avoidance are two known solutions which address the above problem. In congestion control [2], system controls the network parameters after realizing congestion (reactive); whereas, in congestion avoidance, system controls the network parameters before congestion (proactive). After the invention of TCP, there is numerous congestion control algorithms discovered for different purposes. Each of them has unique characteristics [4]. In [5][6] the simulation result shows the TCP BIC giving good throughput for long distance wireless networks, but the TCP Vegas giving better result in the overall performance..

\section{1) Binary Increase Congestion Control (BIC)}

BIC-TCP (Binary Increase Control-TCP) incorporated binary search increase in the protocol. Binary search increase provides reliable feedback on any network congestion and lost packets, allowing BIC-TCP to aggressively increase its transmission speed toward the maximum allowed by the highspeed network. Binary Increase congestion Control for TCP v2.0 is called as CUBIC and it is a default TCP algorithm in Linux.

\section{2) TCP VEGAS}

Until the mid 1990s, all TCPs set timeouts and measured round-trip delays were based upon only the last transmitted packet in the transmit buffer. In TCP Vegas, timeouts were set and round-trip delays were measured for every packet in the transmit buffer. In addition, TCP Vegas uses additive increases in the congestion window.

\section{3) SCALABLE TCP}

Scalable TCP (STCP) involves a simple sender-side alteration to the standard TCP congestion window update algorithm. It robustly improves performance in high-speed, wide-area networks using traditional TCP receivers. Scalable TCP updates its congestion window using fixed increase and decrease parameters.

The Scalable TCP window update algorithm, as defined in [7], is divided into two phases.

Slow-start phase: in which the congestion window is increased by one packet for each acknowledgment received:

$$
\mathrm{W}=\mathrm{W}+1 \text { Ack; }
$$

Congestion avoidance phase: If congestion has not been detected in at least one round trip time, the window responds to each acknowledgment received with the update

$$
\mathrm{W}=\mathrm{W}+\alpha,
$$

Where $\alpha €(0,1)$ is a constant parameter. In the event of congestion, the congestion window is multiplicatively decreased as follows:

$$
\mathrm{W}=\beta \cdot \mathrm{W},
$$

Where $\beta €(0,1)$ is also constant. Typical values of these parameters are $\alpha=0.01$ and $\beta=0.875$. Further details on the Scalable TCP algorithm are available in [9].

\section{The Simulation}

In this paper, these algorithms has been successfully implemented and evaluated using NS-2 simulator on a computer with Intel Core 2 Duo CPU (T6400 processor @ 2.00 $\mathrm{GHz}) 2 \mathrm{~GB}$ of RAM.

A random wireless mobile ad hoc network topology was used for these experiments.

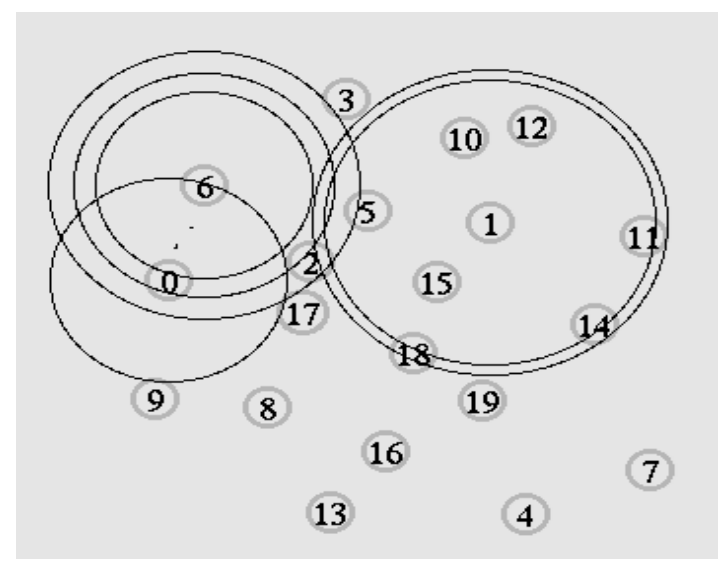

Figure 1. The MANET Scenario

Some of the important parameters of the Ad hoc Network simulation are:

$\begin{array}{ll}\begin{array}{ll}\text { Number of Nodes } \\ \text { Number of Sending Nodes }\end{array} & 1 \\ \text { Topography } & \mathrm{x}=500 \mathrm{y}=500 \\ \text { Mobility } & 0 \text { or } 20 \mathrm{~m} / \mathrm{s} \\ \text { Mobility Start Time } & 20^{\text {th }} \mathrm{Sec} \\ \text { Routing Protocol } & \text { AODV } \\ \text { Mac Type } & 802.11 \\ \text { Queue } & \text { DropTail / PriQueue } \\ \text { Queue Size } & 50 \\ \text { The Traffic Application } & \text { FTP } \\ \text { TCP Packet Size } & 1448 \\ \text { TCP Initial Window Size } & 30000\end{array}$

As far as the different parameters of congestion algorithm are concerned, all default parameters of TCP-Linux have been used in all our simulations. For simplicity and clarity of outputs, we used only one TCP flow during evaluating the algorithms. 


\section{Result And Discussion}

\section{A. Simulation Results in MANETs}

In this section, we carried out the simulation results of congestion control window, Round Trip Delay Time and the Throughput in the Wireless Ad hoc Network without nodes movement and the nodes movement after $20 \mathrm{~m} / \mathrm{s}$. This simulation has been run for 200 seconds.

1) Control Window in MANETs

In the experimental network that we have used to perform experiments for congestion control window comparison between the three algorithms as shown in Figure 2 and Figure 3.

Figure 2 shows the congestion control window increasing and decreasing in all algorithms without any similarities except TCP Vegas.

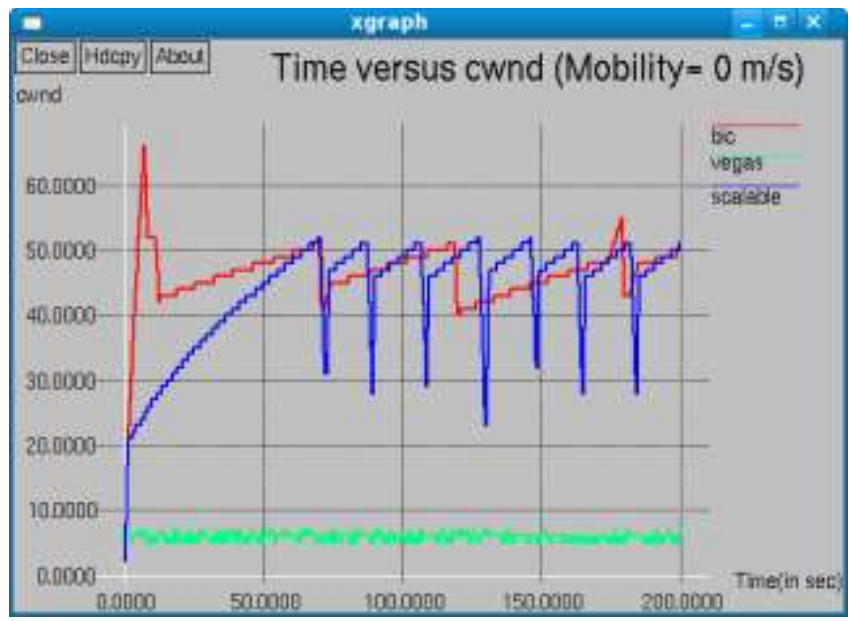

Figure 2. The cwnd on Ad hoc Network without nodes movement

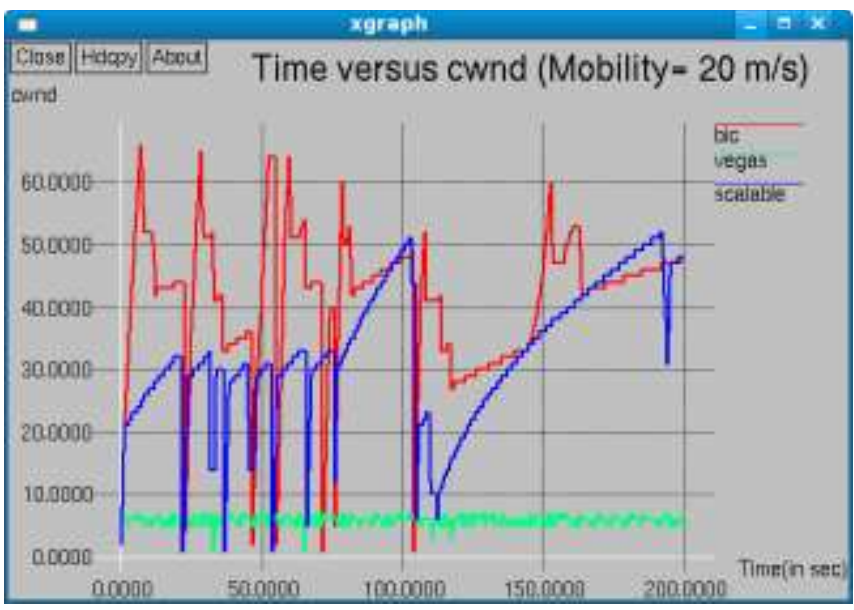

Figure 3. The cwnd on Ad hoc Network nodes movement after $20 \mathrm{~ms}$

In the above Figure 3, the exponential window size increase, linear increase and drop-off occurs irregularly during the simulation. In this Mobile Ad hoc Networks the TCP Vegas giving good result than other algorithms from this group of algorithms. The algorithm Scalable TCP giving second level good performance result in the simulation.

\section{2) Round Trip Delay Time in MANETs}

The Round Trip Delay Time estimation of congestion control algorithms on wireless ad hoc network as shown in the Figure 4 and Figure 5. This simulation result shows the TCP Vegas performance is better than other algorithms. But, the algorithm Scalable TCP is the second highest in this simulation result.

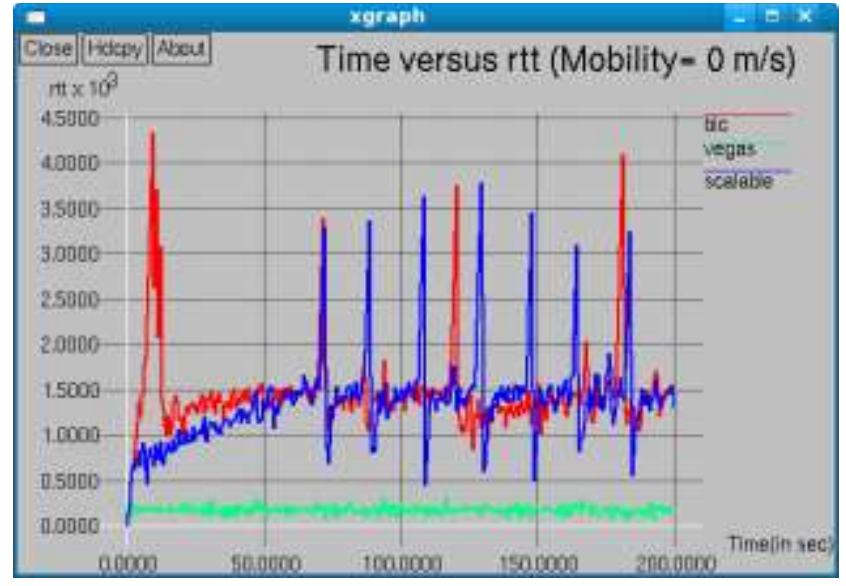

Figure 4. The rtt on Ad hoc Network without mobility

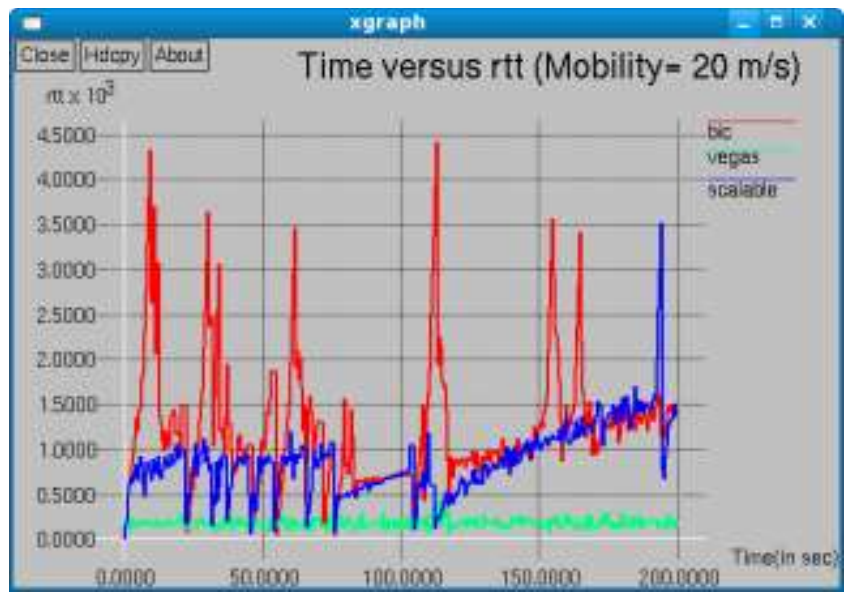

Figure 5. The rtt on Ad hoc Network, the nodes mobility after $20 \mathrm{~m} / \mathrm{s}$

\section{3) Throughput Over Time in MANETs}

Throughput is the average rate of successful message delivery over a communication channel to the destination node. This data may be delivered over a physical or logical link, or pass through a certain network node. The throughput is usually measured in bits per second (bit/s or bps), and sometimes in data packets per second or data packets per time slot.

In this simulation, the throughput is the number of packets reaching to the destination node per ms/second. Here we find out the instant throughput over time.

Figure 6 shows the throughput over time in the non movement ( $0 \mathrm{~ms}$ or no mobility) duration. As per the simulation setup after 50 seconds, all algorithms provided equal performance. During the initial stage, TCP BIC given very low throughput; but over time, all algorithms performed well. 
The Figure 7 shows the throughput over time in the case of nodes movement environment. In this network scenario, all nodes started to move after the time period of 20 seconds in the simulation. As per the simulation result the algorithm Scalable TCP performance was better than other algorithms.

If we carefully observe the two sections (up to 50 seconds and 100 to 200 seconds) of Figure 7, we can say that Scalable TCP throughput performance is better than other algorithms. The algorithm Vegas tried to give better result in the time duration of 20 to 50 seconds. But in the overall time duration Scalable TCP performance was better than other algorithms. So as a final outcome, we selected Scalable TCP is the best performer in mobile ad hoc network scenario to long distance networks. As per the results, we can say the Vegas can be used for short distance communication applications in the less time duration of TCP communication applications.

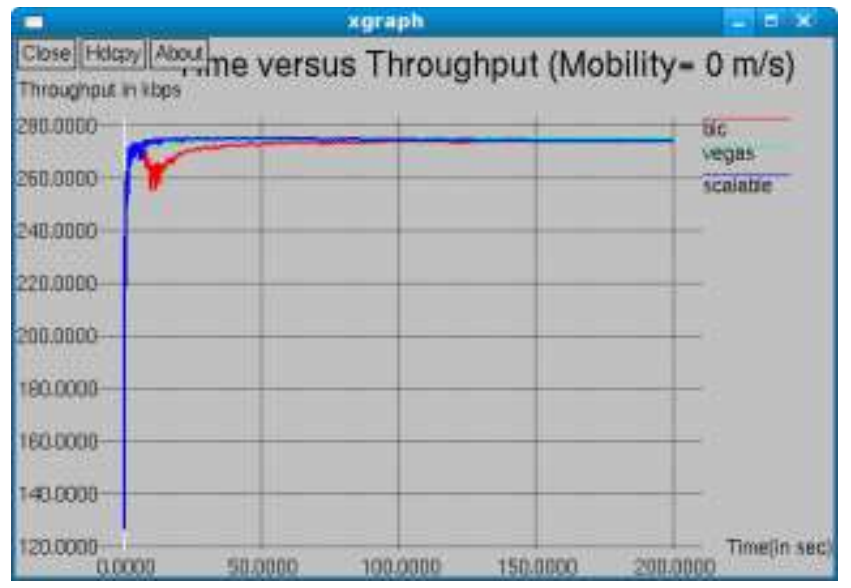

Figure 6. Throughput without Mobility

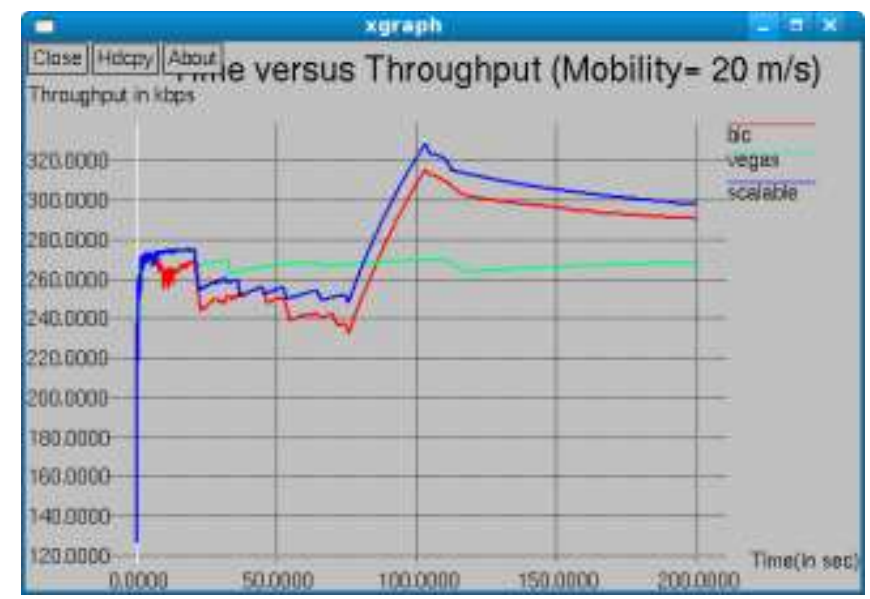

Figure 7. Throughput with Mobility

\section{CONCLUSION}

In this paper we have outlined our implementation and deployment experiences with BIC, Vegas and Scalable TCP in ad hoc network environment. Our experiments have provided the performance of these congestion algorithms in very ideal condition without any cross traffic and any additional flows. In this small MANET scenario, the algorithm Scalable TCP provided good throughput than other algorithms. Algorithm
Vegas performance is better in the control window and RTT. But in the throughput measurement point of view TCP Vegas performance is not good for long distance networks.

Except Scalable TCP, all other assessed algorithms provided low throughput in this simulation. So we conclude Scalable TCP will be the better algorithm for high throughput to long distance data transmission in MANETs.

\section{FUTURE WORK}

As a further work, we have considered to do the improvement on throughput and few more extension in Scalable TCP for better performance. Based on the results, we would extend the future enhancement towards specific application on MANETs.

\section{ACKNOWLEDGMENT}

The authors thank the authorities of the Dr.G.R. Damodharan College of Science, Coimbatore, India, who provided opportunities and resources for carrying out this research work and for the research activities in the Department of Computer Science at the College. The first author further thanks the Management and the Principal of the D.J. Academy for Managerial Excellence, Coimbatore, India for their support and encouragement extended to him to pursue research in the chosen field of study.

\section{REFERENCES}

[1] D. X. Wei and P. Cao, "NS-2 TCP- Linux: An NS-2 TCP Implementation with Congestion Control Algorithms from Linux", proceedings of ValueTool'06 -- Workshop of NS-2, Oct, 2006.

[2] S. Ryu, C. Rump, and C. Qiao, "Advances in Internet congestion control," IEEE Communications Surveys and Tutorials, vol. 3, pp. 28 39, 2003.

[3] Shin-Jer Yang , Yung-Chieh Lin, Soochow University, Taipei, Taiwan, "Tuning Rules in TCP Congestion Control on the Mobile Ad Hoc Networks", Proceedings of the 20th International Conference on Advanced Information Networking and Applications, Volume 01, 2006.

[4] M.Jehan, G.Radhamani, T.Kalakumari, "A survey on congestion control algorithms in wired and wireless networks", Proceedings of the International conference on mathematical computing and management (ICMCM 2010), Kerala, India, June 2010.

[5] M.Jehan, G.Radhamani, T.Kalakumari, "Experimental Evaluation of TCP BIC and Vegas in MANETs", International Journal of Computer Applications (0975-8887), Volume 16-No.1, pp.34-38, February 2011.

[6] M.Jehan, G.Radhamani, T.Kalakumari, “VEGAS: Better Performance Than Other TCP Congestion Control Algorithms on MANETs", International Journal of Computer Networks (IJCN), Volume 3, Issue 2, pp.151-158, May 2011.

[7] Christian Lochert, Björn Scheuermann, Martin Mauve, "A survey on congestion control for mobile ad hoc networks", Wireless Communications \& Mobile Computing, Volume 7 , Issue 5, pp. 655 676, June 2007, ISSN:1530-8669.

[8] M.Allman, V.Paxson, and W.Stevens. TCP Congestion Control. RFC2581 (Proposed Standard), Apr.1999. Updated by RFC3390.

[9] Kelly T, Scalable TCP: Improving Performance in Highspeed Wide Area Networks, 2002, available at http://wwwlce.eng.cam.ac.uk/ ctk21/scalable/.

[10] I. Chlamtac, M. Conti, and J. Liu, "Mobile ad hoc networking: imperatives and challenges", Ad Hoc Networks Journal, vol.1, no. 1, pp. 13-64, Jul. 2003.

[11] A. Al Hanbali, E. Altman, P. Nain, "A Survey of TCP over Mobile Ad Hoc Networks", Research Report no. 5182, INRIA Sophia Antipolis research unit, May 2004. 
[12] Brakmo, L. S., O’Malley, S.W., and Peterson, L.,"TCP Vegas: End-to end congestion avoidance on a global Internet", IEEE Journal on Selected Areas in Communications (JSAC), 13(8), (1995), pp. 14651480.

[13] Mascolo, S. "Congestion control in high-speed communication networks", Automatica, Special Issue on Control Methods for Communication Networks, Vol. 35, no. 12, Dec. 1999, pp. 1921-1935.

[14] Sally Floyd and Kevin Fall, "Promoting the use of end-to end Congestion control in the Internet," IEEE/ACM Transactions on Networking, vol. 7(4), pp. 458-472, August 1999.

[15] Chandrasekaran M. and Wahida Banu R.S.D., "Interaction Between Polynomial Congestion Control algorithms Queue Management schemes in wired TCP Networks," International Journal of Soft Computing, Vol. 1, No. 2, pp.83-90, 2006.

[16] K. Chandran, S. Raghunathan, S. Venkatesan, and R. Prakash," "A feedback based scheme for improving TCP performance in Ad-Hoc wireless networks", Proceedings of the International Conference on Distributed Computing Systems (ICDCS 98), Amsterdam, Netherlands, May 1998.

[17] Foez ahmed, Sateesh Kumar Pradhan, Nayeema Islam , and Sumon Kumar Debnath, " Performance Evaluation of TCP over Mobile Ad-hoc Networks" in (IJCSIS) International Journal of Computer Science and Information Security, Vol. 7, No. 1, 2010.

[18] Mascolo, S., Casetti, C., Gerla, M., Sanadidi, M., Wang. R. "TCP Westwood: End-to-End Bandwidth Estimation for Efficient Transport over Wired and Wireless Networks", In the Proceedings of ACM Mobicom 2001, (Rome, Italy, July 2001).

[19] Chandrasekaran M, Kalpana M and Wahida Banu R.S.D., "Congestion Control using Polynomial Window size adjustment Algorithms for wired and wireless networks", In the Proceedings of International Conference on Network -ICN06 conducted at Mauritius, April 2006.

[20] Chandrasekaran M, , Kalpana M and Wahida Banu R.S.D., "Interaction between MIMD-Poly \& PIPD-Poly Algorithms and other TCP Variants in Multiple Bottleneck TCP Networks", In the Proceedings of IEEE Conference WOCN2006, Bangalore, April 2006.

[21] Tomoya Hatano, Hiroshi Shigeno, Ken-ichi Okada, "TCP-friendly Congestion Control for High Speed Network", International Symposium on Applications and the Internet- SAINT'07, pp.10, 2007.

[22] David X. Wei, Cheng Jin, Steven H. Low, and Sanjay Hedge., "Fast TCP: Motivation, Architecture, Algorithms, Performance", IEEE/ACM transactions on networking, 2006.
[23] K. Satyanarayan Reddy and Lokanatha C. Reddy., "A survey on congestion control mechanisms in high speed networks", IJCSNSInternational Journal of Computer Science and Network Security, vol. 8, no. 1,2008 , pp. $187-195$.

[24] Colin Perkins and Ladan Gharal., "Rtp and the datagram congestion control protocol", In Proceedings of .IEEE International Conference on Multimedia and Expo, Toronto, Canada, July 2006.

[25] Van Jacobson., "Congestion Avoidance and Control", Computer Communications Review, Volume 18 number 4, pp. 314-329, August 1988.

[26] Van Jacobson., "Modified TCP Congestion Control Avoidance Algorithm", end-2-end-interest mailing list,pp.1-14 April 30, 1990.

[27] A Linux TCP implementation for NS-2. URL: http://www.cs.caltech.edu/weixl/ns2.html

[28] A mini - tutorial for NS-2 TCP-Linux. URL: http://www.cs.caltech.edu/weixl/ns2.html

\section{AUTHORS PROFILE}

M.Jehan is an Associate Professor of Computer Science Department at D. J. Academy for Managerial Excellence, Coimbatore, India. He received his B.Sc degree in Computer Science from Manonmaniam Sundaranar University in 1998 and the M.Sc degree in Computer Science from Bharathidasan University, Tiruchirappalli, India in 2000. He completed him M.Phil degree under Manonmaniam Sundaranar University, Tirunelveli, India in 2003. He is doing him Ph.D in Mobile Computing at Dr.G.R.Damodaran College of Science, Coimbatore under Bharathiar University, India. He has published more number of papers in International Journals and Conferences. His research interests are Wireless Networks, Congestion Control and Ad hoc Networks.

Dr.G.Radhamani has over 20 years of experience in teaching and research, working as Professor and Director, Department of Computer Science, Dr. G.R.Damodaran College of Science, India. She did her PDF (Post Doctoral Fellow) in the Department of Computer Science and Engineering from IIT Chennai. She received her $\mathrm{PhD}$ (Computer Engineering) from Multimedia University, Malaysia and M.Sc., M.Phil (Computer Science) degrees from PSG College of Technology, India. She served in Multimedia University, Malaysia from August 2001 to May 2006. She has published more number of papers in International Journals and Conferences. She is a Senior Member of IEEE and CSI. Her research interests are Databases, Computer Security and Mobile Computing. 